\title{
Contextual Interference Effect in Bandwidth and Self-Control Feedback Conditions on Relative and Absolute Timing Learning
}

\author{
${ }^{1}$ Amir Shams ${ }^{*}{ }^{2}$ Seyedeh Mansoreh Naeimi Tajdar \\ ${ }^{1}$ Sport Sciences Research Institute, Iranian Ministry of Sciences, Research and Technology, Tehran, Iran. ${ }^{2}$ Department \\ of Motor Behavior, Faculty of Sport Sciences, Central Tehran Branch, Islamic Azad University, Tehran, Iran.
}

\begin{abstract}
This study aims to better understand the effect of practice schedule and feedback providing types. In two separate experiments the contextual interference effect in bandwidth and self-control feedback conditions on relative and absolute timing learning was examined. In experiment $\mathrm{I}$, the effect of contextual interference using bandwidth and self-control feedback on absolute timing learning (parameters) was studied. Results in the acquisition test showed the performance of blocked self-control and bandwidth groups were significantly better than other groups $(\mathrm{P}<0.05)$. There was no significant difference in retention and transfer tests $(\mathrm{P}>0.05)$. In experiment II, the effects of contextual interference with using a bandwidth and self-control feedback on relative timing learning (GMP) were studied. . In retention and transfer tests, the performance of random self-control and bandwidth groups was significantly different to the other groups $(\mathrm{P}<0.05)$. The performance of self-control feedback groups in both experiments was better than bandwidth feedback groups $(\mathrm{P}<0.05)$ in all stages. The results of this study reveal the beneficial effect of self-control feedback on relative timing (GMP) rather than absolute timing (parameter) learning and supported the theoretical separation of this processes.
\end{abstract}

KEY WORDS: Bandwidth Feedback, Self-Control Feedback, Absolute Timing, Relative Timing, Contextual Interference.

\section{INTRODUCTION}

Motor program is located in the heart of theories based on central control. Schmidt (1985, 1975) proposed that programmed actions are controlled by the generalized motor programs (GMPs) that provide a common structure to a class of actions. Independent roles or schemata (recall schema) provided the scaling features for generalized motor programs, so that specific actions can be generated. When errors of previous tasks were detected, recognition schema provides the mechanisms for evaluating and regulating recall schema before the next task $(1,2)$. Accordingly, the GMP developed during exercise underpins the production of a class of tasks that have fixed features (such as sequencing, relative timing and relative force(.On the other hand, specific tasks are created by pre-movement scaling factors (known as parameters) such as absolute force or absolute timing through recall schema. According to Schmidt (1985) relative timing is a measure underlying the movement structure or GMP while absolute timing is a measure of the

*. Corresponding Author:

Amir Shams

E-mail: amirshams85@gmail.com 
capability to parameterize an action appropriately (2).

Thus, Schmidt (2011) stated theoretical constructions of GMP and parameters which affect the programmed actions are controlled by a separate memory. On this basis, researchers in the past have claimed that learning of GMP and parameter are two separate processes and based on these findings have proposed dissociation of GMP from the parameter (3). According to this theory, the theoretical structures of GMP and parameterization processes are separated.

Also, Shea et al. (2001) have claimed that in the future, research is needed to determine the factors (such as practice schedules and types of feedback providing) in order to reach the optimal levels of generalized motor program and parameter learning (4). Recently, researchers have attempted to study the GMP and its associated parameters through practice. One of the major research lines in this field that can provide evidence related to theoretical dissociation between the GMP and parameterized processes, is manipulating the practice schedule or contextual interference (CI) (5). CI is defined as interference in performance and learning that creates from practice, one task in the context of other tasks (3). CI is located on a continuum in which the blocked practice referred to as low interference, and random practice referred as high interference (6). The origin of CI is derived from Battig $(1966,1972)$ that has done in the field of verbal learning. Battig, in their initial investigation found that factors causing difficulty for the learner in the acquisition phase, impairs their performance in the acquisition phase, but will improve performance during retention and transfer tests (7). Magill (2011) also believes that this effect can be explained by contextual dependency. Accordingly, the practice with low CI (blocked practice) creates a dependency on the practice field (6). This dependency makes comprehensive response capability in the change of the task or practice does not improve rather than high CI (random practice).

Wulf, Schmidt, and Deubel (1993) also proposed that the effect of practice variables on the learning process is complex, but that these variables are very dependent on the nature of the task and the level of the individual's experience
(8). Some of these complexities have recently become the theoretical framework by Guadagnoli and Lee (2004). They proposed that cognitive processing during the practice period depends on the rate of challenge during the practice. The nature of the task, practice schedules and learner skill levels interact together to determine the level of challenge in the practice trials (9). Guadagnoli, Holcomb, and Weber (1999) noted that high CI at the beginning of practice may not always be useful for learning. Rather, the increased CI in the beginning stage of practice led to overwhelmed responses and reduced learner performance in the retention and transfer tests. These results suggest that when the learner encounters challenging tasks, incompetency in the information-processing system leads to unclear interpretation of the required information. Finally, this will lead to a lack of individual learning (10).

Another factor that can affect the performance quality and motor skill learning process is feedback which provides evidence in relation to the separate dissociation of GMP and parameterization processes. In this regard, the results of various studies show that decreasing the knowledge of result (KR) frequency has an important effect on motor programs learning, and that this factor can cause the response stability and therefore lead to improvement of GMP and parameter (11). In this regard, bandwidth feedback is one of the methods for reducing the feedback frequency and coaches or researchers based on the pre-determined range use this feedback type about the performance goal. Goodwin and Meeuwsen (1995) applied bandwidth feedback in the sports field with a golf putting skill. In this study, there were four bandwidth feedback schedules including a $0 \%$ bandwidth (feedback provided in every trial), $10 \%$ bandwidth, shrinking bandwidth (increasing amounts of feedback) and expanding bandwidth (decreasing levels of feedback). Their results showed that both the $10 \%$ and expanding bandwidth groups performed significantly higher on the retention test (12). In addition, Lai and Shea (1999) showed that the constant and serial practice of using bandwidth feedback affected absolute timing learning (13). Lai et al. (2000) also noted that bandwidth feedback is effective 
in relative timing in relation to absolute timing learning (14).

One other method for reduced KR frequency (that coaches always neglect) is self-control feedback. Chiviacowsky et al. (2008) stated that self-control feedback refers to a type of KR in which the learner actively chooses when to receive information about the outcome of his/her performance or score (15). Based on their results, Zimmerman (1996, 1989), Chen and Singer (1992) and Chiviacowsky and Wulf (2005) suggest that the perception of self-control enhances learning, leading to more active involvement of learners in the performance and learning process. Thus, giving the learner control over practice sessions might promote a deeper level of processing of relevant information (1619). Studies done on the effect of self-control feedback on relative and absolute timing reveal inconsistent results. Chiviacowsky and Wulf (2002) revealed that self- control feedback could affect absolute timing learning (20). However, in another study Chiviacowsky and Wulf (2005) reported that self- control feedback is affective in relative timing feedback in relation to the absolute timing (17).

In relation to effectiveness high CI, Wulf \& Shea (2004), Wright and Shea (2001) and Wulf, Schmidt, and Deubel (1993) reported that random practice, with reduced KR frequency, has a greater effect on learning of relative timing rather than absolute timing $(8,11,21)$. In this study, two separate experiments were conducted in order to examine the contextual interference effect in bandwidth and self-control feedback conditions on relative and absolute timing learning.

\section{MATERIALS AND METHODS}

Participants. In Experiment I, ninety righthanded students with no previous experience of the related task were selected and randomly divided into 6 groups (including blocked, serial and random groups in self-control and bandwidth feedback conditions). In Experiment II, ninety right-handed students with no previous experience of the related task were used in the study and randomly divided into 6 groups of 15 persons (Including blocked, serial and random practice groups in self-control and bandwidth feedback conditions).
Measurement tools and procedure. In Experiment I, We used the rotary pursuit system (Model A. 30014) manufactured by American Lafayette systems to measure hand-eye coordination and consists of two parts (software and hardware). Using this device, the parameters (different speeds) and the GMPs (different models) can be evaluated. For the task, subjects were placed behind the system and with the start signal given by computer, a follow-able light starts to turn, and the subject should follow the light by the lever that is connected to the device. Moreover, Time off Target (TOT) was recorded as an error of subjects by the computer and the performance score of subjects was calculated as Root Mean Square Error (RMSE) for each experiment.

The task given to subjects in experiment I was to pursue the light target on the rotary pursuit system with constant GMP (quadrangle) and variable parameters (with speeds of 20, 30 and 40 cycles per minute). The pre-test included one block of $9(3 \times 3)$ trials, whereby subjects performed 3 trials in each speed parameter. In acquisition phase, the subjects participate in the 8 sessions in 8 days. In each session, subjects performed two blocks of 9 trials which included three trials in each speed parameter.

For each subject in bandwidth feedback groups, the time error of 20 seconds for each trial determined as a performance error that based on this range the feedback provided for them. In addition, in this period, the self-control feedback was requested by subjects and providing feedback frequency (33 percent) was similar to all self-control groups. After the latest practice trials, the acquisition test was done. After $72 \mathrm{hrs}$ the subjects participated in a retention test and performed one block of 9 trials similar to the pretest period trials. After the retention test, the transfer test in each experiment was done separately. The speed of 50 cycles per minute (new parameter) consisted as a transfer task that the subjects performed this task in one block of 9 trials.

In Experiment II, Tools used in this experiment were similar to Experiment I.

The task of subjects in experiment II was to pursue light target on the rotary pursuit system with constant parameter (with speed of 20 cycles per minute) and variable GMPs (circle, 
quadrangle and triangle). The pre-test included the one block of 9 trials $(3 \times 3)$, that subjects performed 3 trials in each GMPs. In acquisition phase, the subjects participate in the 8 sessions in 8 days. In each session, subjects performed two blocks of 9 trials that included three trials in each GMP.

For each subject in bandwidth feedback groups, the time error of 20 seconds for each trial determined as a performance error that based on this range the feedback provided for them. Furthermore, in this period, the self- control feedback based on subjects requests provided feedback for them, and the providing feedback frequency (33 percent) was similar to all selfcontrol groups. After the latest practice trials, the acquisition test was done. After 72 hrs the subjects participated in retention test and performed one block of 9 trials similar to the pretest period trials. After the retention test, the transfer test was done. The diamond pattern (new GMP) was consisted as a transfer task that the subjects performed this task in one block of 9 trials.

Statistical Analysis. For data analysis, the descriptive statistics (such as mean and standard deviation) and inferential statistics such as oneway analysis of variance (ANOVA) test in pretest and two-factorial analysis of variance 3 (type of practice or groups) $\times 2$ (feedback) in acquisition, retention and transfer tests were used. Furthermore, in order to identify the differences amongst groups in both experiments, the Bonferroni Post hoc test was used. For data analysis we used SPSS version 15, and for drawing graphs the Excel 2007 program were used.

\section{RESULTS \\ Experiment I}

Pre-test. One-way ANOVA test results showed the difference of mean scores of subjects in first test $\left(\mathrm{F}_{(5,84)}=0.498\right.$ and $\left.\mathrm{P}=0.813\right)$ is not significant.

Acquisition test results. Two-factorial analysis of variance results showed the main effects of the practice schedule with $\mathrm{F}=44.558$ was significant at the level of $P=0.001$. The Bonferroni Post hoc test results showed that there was a significant difference between block practice groups and serial/random groups. The interaction effects of feedback type and practice schedule with $F=7.53$ was significant $(\mathrm{P}<0.05)$. Bonferroni Post hoc test results showed that performance of blacked bandwidth and self-control feedback groups was significantly different from other groups. Also, the main effect of feedback type with $\mathrm{F}=126.03$ at the level of $\mathrm{P}<0.05$ was significant. Generally, the self-control feedback group (=6.07) had fewer performance errors than the bandwidth feedback group $(=6.28)$. Graph 1 shows the absolute timing error scores in experiment $\mathrm{I}$, in all test phases.

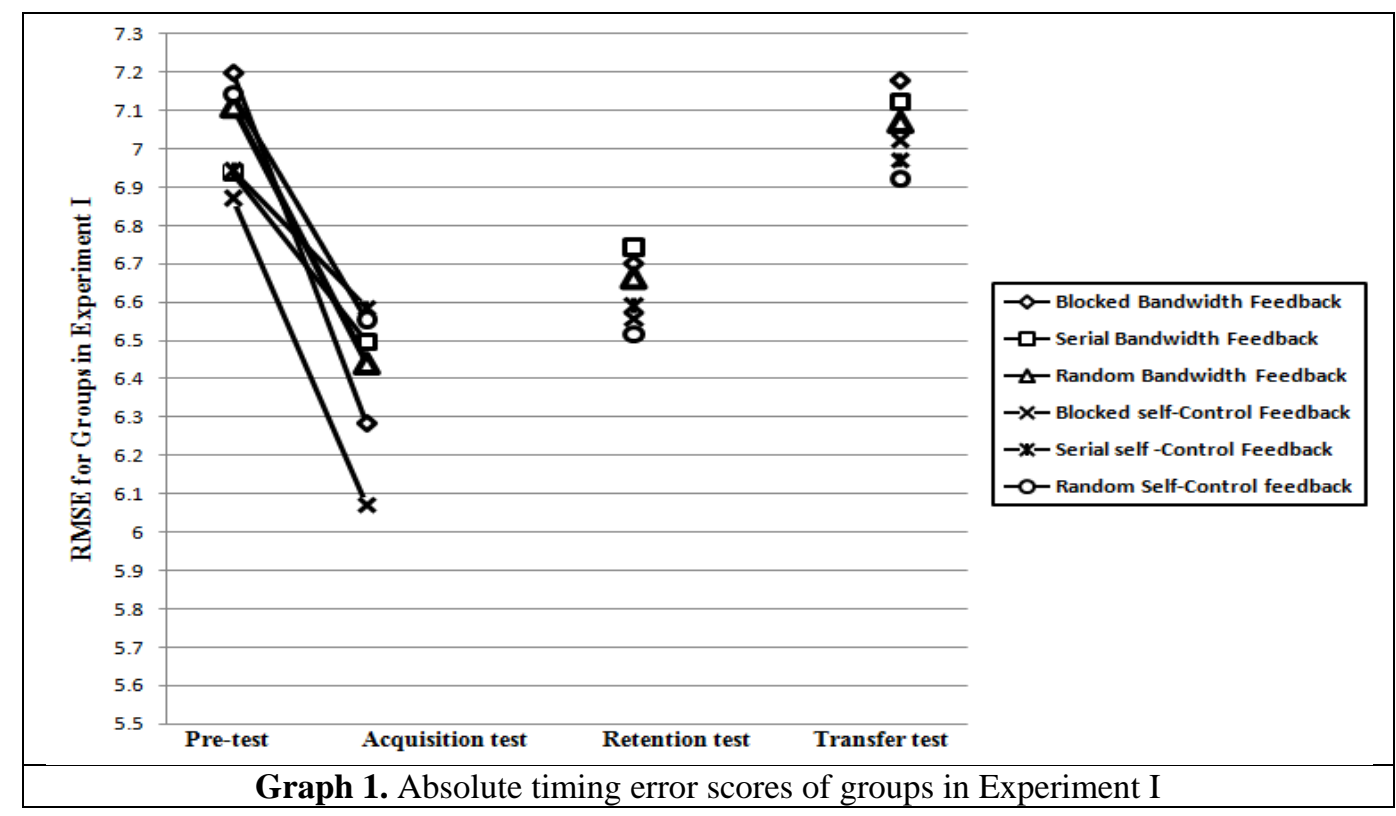

Shams, A., Naeimi, S. M. Tajdar. (2016). Ann Appl Sport Sci, 4(2): 49-57. 
Retention test results. Two-factorial analysis of variance showed that the main effect of practice schedule with $\mathrm{F}=1.507$ and the interaction effect of feedback type and practice schedule with $\mathrm{F}=0.814$ in the level of $\mathrm{P}>0.05$ was not significant, while the main effect of feedback type with $\mathrm{F}=43.640$ in the level of $\mathrm{P}<0.05$ was significant. Generally, the selfcontrol feedback group $(=6.55)$ had less performance errors than the bandwidth feedback group (=6.74).

Transfer test results. Two-factorial analysis of variance results showed that main effect of practice schedule with $\mathrm{F}=0.071$ and interaction effect of feedback type and practice schedule with $\mathrm{F}=0.799$ in the level of $\mathrm{P}>0.05$ was not significant, while the main effect of feedback type with $\mathrm{F}=84.474$ at the level of $\mathrm{P}<0.05$ was significant. The results show that the self-control feedback group $(=7.03)$ had less performance errors than the bandwidth feedback group $(=7.19)$.

\section{Experiment II}

Pre-test. One-way ANOVA test results showed that difference of mean scores of subjects in this experiment with $\mathrm{F}_{(5,84)}=0.375$ at the level of $\mathrm{P}=0.864$ was not significant.

Acquisition test results. Two-factorial analysis of variance results showed that the main effect of practice schedule with $\mathrm{F}=22.896$ was significant at the level of $\mathrm{P}<0.05$.The Bonferroni Post hoc test results showed that there was a significant difference between blocked practice groups with serial and random groups. The interaction effect of feedback type and practice schedule with $\mathrm{F}=0.19$ was not significant $(\mathrm{P}>$ 0.05). Also, the main effect of feedback type with $\mathrm{F}=33.413$ was significant at the level of $\mathrm{P}<$ 0.05 . Generally, self- control feedback group (=6.10) had fewer performance errors than the bandwidth feedback group (=6.39). Graph 2 shows relative timing error scores in experiment II for all test phases.

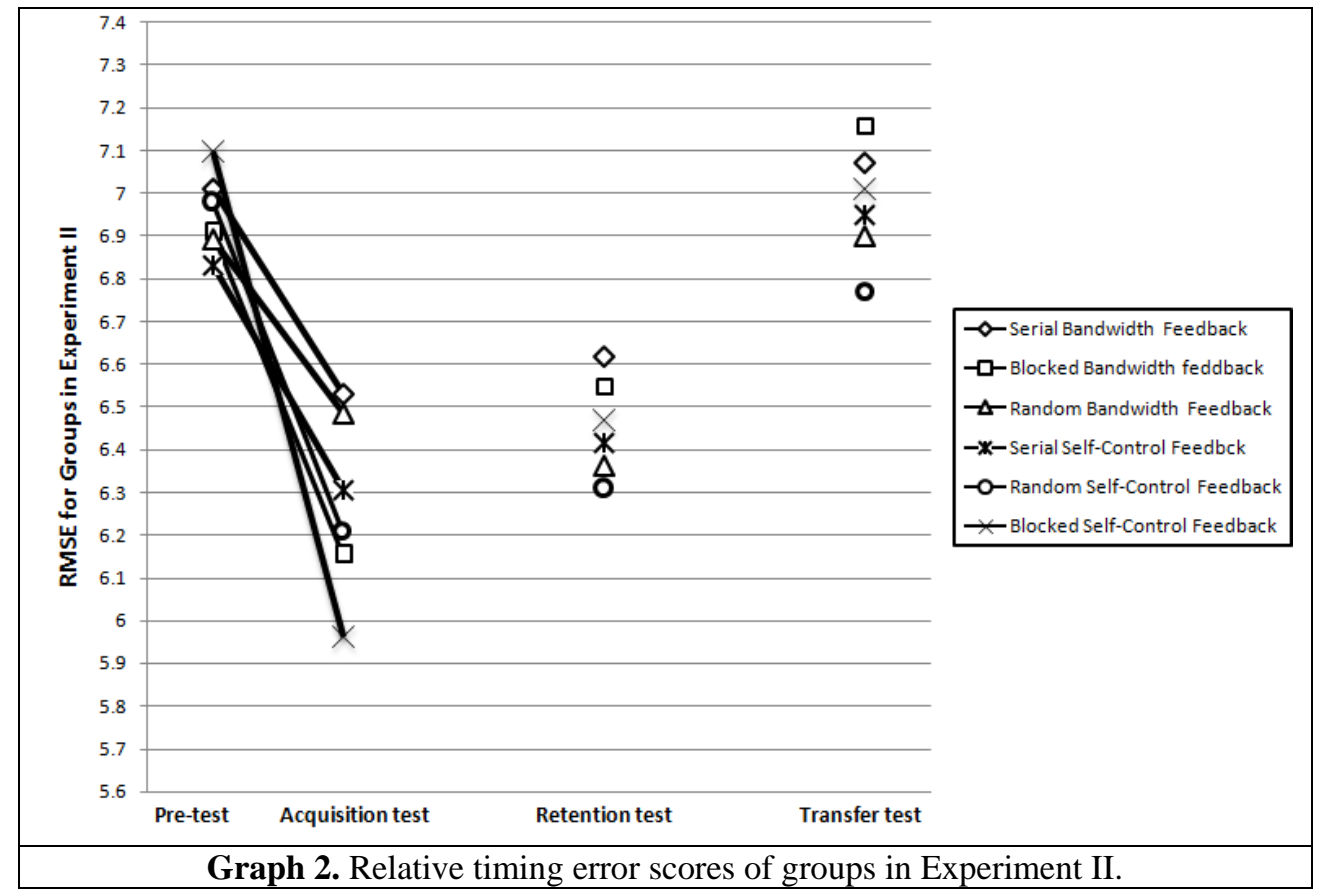

Retention test results. Two-factorial analysis of variance showed that the main effect of practice schedule with $\mathrm{F}=31.867$ at the level of $\mathrm{P}<0.05$ was significant. The Bonferroni Post hoc test results showed that there was a significant difference between random practice groups with serial and random groups. The main effect of feedback type with $\mathrm{F}=22.202$ at the level of $\mathrm{P}<0.05$ was significant. The result showed that the self- control feedback group 
(=6.29) had fewer performance errors than the bandwidth feedback group (=6.49). Moreover, the interaction effect of feedback type and practice schedule with $\mathrm{F}=1.601$ in the level of $\mathrm{P}>0.05$ was not significant.

Transfer test results. Two-factorial analysis of variance showed that the main effect of practice schedule with $\mathrm{F}=57.457$ at the level of $\mathrm{P}<0.05$ was significant. The Bonferroni Post hoc test results showed that there was a significant difference between random practice groups with serial and random groups. The main effect of feedback type with $\mathrm{F}=31.584$ at the level of $\mathrm{P}<0.05$ was significant. The result showed that the self- control feedback group $(=6.90)$ has less performance errors than the bandwidth feedback group (= 7.04). Moreover, the interaction effect of feedback type and practice schedules with $\mathrm{F}=$ 0.124 in the level of $\mathrm{P}>0.05$ was not significant.

\section{DISCUSSION}

\section{Experiment I}

The results obtained from the acquisition test shows better performance of blocked selfcontrol and bandwidth feedback groups in relation to serial and random self-control and bandwidth feedback groups. Also, the performance groups in retention and transfer test was not significant. These results in the acquisition phase are consistent with the findings of Maslovat et al. (2004) and Meira and Tani (2001) $(22,23)$. The results of this part are inconsistent with the findings of Jones and French (2007) and Zetou et al. (2007) (24, 25). These researchers interpreted the results of their research based on the task's type. They stated that perhaps not causes the CI effect assignments to the field studies (volleyball skills) attributed to the laboratory researches. Results in retention and transfer parameters are consistent with the findings of Lee, Wulf, and Schmidt (1992) (26). They believed that CI has no effect on retention and transfer parameters and stated that to enhance learning of motor skills, the high levels of CI which are control by different GMPs, was effective (26). But this result is inconsistent with the findings of Sekiya and Magill (2000) and Vera and Montilla $(2003)(27,28)$. They noted that one of the main factors to create the CI is the amount of practice. In this study subjects in the acquisition phase was performed in 16 blocks of nine trials totaling 144 trials. Perhaps one of the reasons that CI effect was not observed in the retention and transfer parameter is related to this agent, and it is proposed that in future studies, researchers should consider these factors in their researches.

Chiviacowsky and Wulf (2005) noted that reducing the frequency of feedback will reduce the absolute timing of learning (17), and this result is consistent with our study results. So, the present results support the schema theory. The results of our study showed that the effectiveness of self-control feedback rather than bandwidth feedback is consistent with findings of Wulf (2007) and Chiviacowsky and Wulf (2002) (20, 29). They noted that if the learner in the acquisition phase has control over the timing of the feedback given, they will show better performance in the acquisition, retention and transfer phases. Learners will show more comprehensive effort because having control in practice trials acts as a strong internal stimulating factor $(20,29)$.

\section{Experiment II}

The results obtained in the acquisition test shows better performance of blocked selfcontrol and bandwidth feedback groups in relation to the serial and random self-control and bandwidth groups. These results in the acquisition test are consistent with the findings of Grand et al. (2015), Porter et al. (2007), Fairbrother, Shea, and Marzilli (2007) and Magill andHall (1990) (30-33).

The results of the acquisition test are inconsistent with elaboration, and cognitive effort hypothesizes. Based on the elaboration hypothesis, the random practice will create in the learner meaningful, cognitive processing of the learned task. According to this hypothesis, the existent differences in tasks during random practice can improve the analysis for related tasks. In conclusion, representation of each specific task after random practice in relation to the blocked practice will be more in mind. On the other hand, Lee, Wulf, and Schmidt (1992) presented the cognitive effort hypothesis (26). They stated that cognitive effort refers to an amount of mental work during decision making; they also argued that produced adjustments in motor program's modifications, firstly, done as a cognitive model and then with the 
unconsciousness way can be integrated with motor memory; and so, they recommended that random practice increases the cognitive efforts and then improves learning (26).

The results in retention and transfer tests showed that the interaction effect of feedback type and practice arrangement was not significant but the mean effect of feedback type was significant, and the self-control feedback groups have the better performance than bandwidth feedback groups. Also the mean effect of practice schedule was significant and mean performance of the random group was better than other groups. Therefore, the results of experiment II in the retention test were consistent with Boutin and Blandin (2010), Porter et al. (2007) and Magill and Hall (1990) results $(5,32,33)$. Also, the results of this study in transfer test are consistent with Russell and Newell (2008) and Carter and Ste-Marie (2016) results $(34,35)$. Based on their results, GMP transfer in relation to the parameter transfer revealed more interactive effects $(34,35)$; but was inconsistent with Zipp and, Gentile (2010) results (36). The best justification about these results, could be noted the non-laboratory or applied this experiment than our study.

Magill (2011) believed that this effect can be explained by contextual independency (6). Contextual dependency refers to a situation whereby learners develop a dependency on the context in which blocked practice occurs. This then hinders performance during transfer to task variations or conditions different from those experienced in practice rather than random practice (high CI) (6).

The results of experiment II is consistent with Chiviacowsky and Wulf (2005) that showed reduced KR frequency with self-control methods increase the relative timing of learning (17); but is inconsistent with Chiviacowsky and Wulf (2002) results (20). This may be one of the factors responsible for these outcomes was the task type (timing task with pressing the 2, 4, 6 and 8 of computer keys) and type of feedback providing (only for total time of movement)

\section{CONCLUSION}

One of the fundamental issues in motor learning research is to determine the conditions for effective training and providing feedback information that facilitates learning and performance improvement and will be presented to the learner. This study was examining the contextual interference effect in bandwidth and self-control feedback conditions on relative and absolute timing learning. In two separate experiments, the results showed that in the relative timing or GMP) in the retention and transfer tests, random group has better performance than the serial and blocked groups but in the experiment I (absolute timing or parameter), such effect was not observed.

The results of this study support for the Magill \& Hall (1990) hypothesis. Also Cross, Schmitt, and Grafton (2007) (37), while studying neural control regions involved in contextual interference during motor skill learning found that random practice groups in the pre-motor and motor-sensory regions have more activity than the other groups. Given that these two regions are involved in the preparation, sequencing, and selected responses, results from this study support the hypothesis of contextual interference advantage due to improving individual's memory capacity to prepare a motor response. Although, recent studies have shown that the usefulness of self-control feedback on learning motor skills [e.g. Chiviacowsky and Wulf 2005, 2002 and Janelle et al. 1997 (17, 20, 38)] but few studies have been done to clarify relative and absolute timing learning.

Therefore, understanding the affective factors on self-control feedback not only is important theoretically but also leads to the development of practice methods in applied fields. Chiviacowsky et al. (2008) stated that the perception of selfcontrol conditions can improve learning because it leads to more active involvement of the learner in the learning process. Chiviacowsky et al. (2008 and 2008) and Carter, Carlsen, and SteMarie (2014) believed that giving learners the opportunity to control a certain aspect of practice conditions led to successful trials and successful performance strategies for learners $(15,39,40)$. This can improve error detection and reforming them between the efforts that haven't provided any feedback and finally the respond to stability increases.

Therefore, detection and correct error development are the other advantages of skills learning in self-control feedback. Based on this 
study, results in regard to the effectiveness of self- control feedback along the continuum of reduced KR frequency, it seems that practice sessions permitting learners to decide when and why to get feedback increases the effects of the practice. If the learner is actively involved in the learning process it leads to deeper processing of the task. Using the self- control feedback via reduced KR frequency and performance stability in practice efforts leads to development of relative timing (GMP).

Wulf (2007) argued that perception of selfregulation conditions help the learner to increase the feedback effect by using specific strategies such as attention guidance and information transfer (29). Current studies show that selfcontrol feedback has a significant effect on relative timing learning. This different effect of self-control feedback on learning of relative and absolute timing is evidence of the separate dissociation of GMP from parameter learning. The present study shows that the self-control feedback was significantly more affective on learning of relative timing than absolute timing.
Therefore, this present study provides a new approach to study the underlying reasons of learning advantages of using self-control feedback. Finally, based on the results of this study it seems that coaches can apply the high CI (random practice) whilst using self-control feedback.

\section{APPLICABLE REMARKS}

- Based on our results, the self- control feedback along the continuum of reduced KR frequency, are permit the learners to decide when and why to get feedback increases the effects of the practice.

- Based on our results, the self-control learners will show more comprehensive effort because having control in practice trials acts as a strong internal stimulating factor.

\section{REFERENCES}

1. Schmidt RA. A schema theory of discrete motor skill learning. Psychological Review. 1975;82(4):225-60.

2. Schmidt RA. The 1984 C. H. McCloy Research Lecture: The Search for Invariance in Skilled Movement Behavior. Research quarterly for exercise and sport. 1985;56(2):188-200.

3. Schmidt RA, Lee TD. Motor Control and Learning: A Behavioral Emphasis: Human Kinetics; 2011. 581 p.

4. Shea CH, Lai Q, Wright DL, Immink M, Black C. Consistent and variable practice conditions: effects on relative and absolute timing. Journal of motor behavior. 2001;33(2):139-52.

5. Boutin A, Blandin Y. Cognitive underpinnings of contextual interference during motor learning. Acta psychologica. 2010;135(2):233-9.

6. Magill RA. Motor Learning and Control: Concepts and Applications. 9th ed: McGraw-Hill; 2011. 466 p.

7. Battig WF. Intratask interference as a source of facilitation in transfer and retention. In: Thompson RF, Voss T, editors. Topics in learning and performance. New York, USA: Academic Press Inc; 1972.

8. Wulf G, Schmidt RA, Deubel H. Reduced feedback frequency enhances generalized motor program learning but not parameterization learning. Journal of experimental psychology Learning, memory, and cognition. 1993;19(5):1134-50.

9. Guadagnoli MA, Lee TD. Challenge point: a framework for conceptualizing the effects of various practice conditions in motor learning. Journal of motor behavior. 2004;36(2):212-24.

10. Guadagnoli M, Holcomb WR, Weber TJ. The relationship between contextual interference effects and performer expertise on the learning of a putting task. Journal of Human Movement Studies. 1999;37(1):19-36.

11. Wulf G, Shea CH. Understanding the role of augmented feedback: the good, the bad, and the ugly. In: Williams AM, Hodges NJ, editors. Skill Acquisition in Sport: Research, Theory and Practice. London, UK: Taylor \& Francis (Routledge); 2004. p. 121-44.

12. Goodwin JE, Meeuwsen HJ. Using bandwidth knowledge of results to alter relative frequencies during motor skill acquisition. Research quarterly for exercise and sport. 1995;66(2):99-104.

13. Lai Q, Shea CH. Bandwidth Knowledge of Results Enhances Generalized Motor Program Learning. Research quarterly for exercise and sport. 1999;70(1):79-83.

14. Lai Q, Shea CH, Wulf G, Wright DL. Optimizing generalized motor program and parameter learning. Research quarterly for exercise and sport. 2000;71(1):10-24. 
15. Chiviacowsky S, Wulf G, de Medeiros FL, Kaefer A, Tani G. Learning benefits of self-controlled knowledge of results in 10-year-old children. Research quarterly for exercise and sport. 2008;79(3):405-10.

16. Chen D, Singer RN. Self-regulation and cognitive strategies in sport participation. International Journal of Sport Psychology. 1992.

17. Chiviacowsky S, Wulf G. Self-controlled feedback is effective if it is based on the learner's performance. Research quarterly for exercise and sport. 2005;76(1):42-8.

18. Zimmerman BJ. A social cognitive view of self-regulated academic learning. Journal of Educational Psychology. 1989;81(3):329-39.

19.Zimmerman BJ, Kitsantas A. Self-regulated learning of a motoric skill: The role of goal setting and selfmonitoring. Journal of Applied Sport Psychology. 1996;8(1):60-75.

20. Chiviacowsky S, Wulf G. Self-controlled feedback: does it enhance learning because performers get feedback when they need it? Research quarterly for exercise and sport. 2002;73(4):408-15.

21. Wright DL, Shea CH. Manipulating generalized motor program difficulty during blocked and random practice does not affect parameter learning. Research quarterly for exercise and sport. 2001;72(1):32-8.

22. Maslovat D, Chus R, Lee TD, Franks IM. Contextual interference: single task versus multi-task learning. Motor control. 2004;8(2):213-33.

23. Meira CM, Jr., Tani G. The contextual interference effect in acquisition of dart-throwing skill tested on a transfer test with extended trials. Perceptual and motor skills. 2001;92(3 Pt 1):910-8.

24. Jones LL, French KE. Effects of contextual interference on acquisition and retention of three volleyball skills. Perceptual and motor skills. 2007; 105(3 Pt 1):883-90.

25.Zetou E, Michalopoulou M, Giazitzi K, Kioumourtzoglou E. Contextual interference effects in learning volleyball skills. Perceptual and motor skills. 2007;104(3 Pt 1):995-1004.

26. Lee TD, Wulf G, Schmidt RA. Contextual Interference in Motor Learning: Dissociated Effects Due to the Nature of Task Variations. The Quarterly Journal of Experimental Psychology Section A. 1992;44(4):627-44.

27. Sekiya H, Magill RA, Anderson DI. The contextual interference effect in parameter modifications of the same generalized motor program. Research quarterly for exercise and sport. 1996;67(1):59-68.

28. Vera JG, Montilla MM. Practice Schedule and Acquisition, Retention, and Transfer of a Throwing Task in 6YR.-Old Children. Perceptual and motor skills. 2003;96(3):1015-24.

29. Wulf G. Self-controlled practice enhances motor learning: implications for physiotherapy. Physiotherapy. 2007;93(2):96-101.

30. Fairbrother JT, Shea JB, Marzilli TS. Repeated retention testing effects do not generalize to a contextual interference protocol. Research quarterly for exercise and sport. 2007;78(5):465-75.

31. Grand KF, Bruzi AT, Dyke FB, Godwin MM, Leiker AM, Thompson AG, et al. Why self-controlled feedback enhances motor learning: Answers from electroencephalography and indices of motivation. Human Movement Science. 2015;43:23-32.

32. Magill RA, Hall KG. A review of the contextual interference effect in motor skill acquisition. Human Movement Science. 1990;9(3-5):241-89.

33. Porter JM, Landin D, Hebert EP, Baum B. The Effects of Three Levels of Contextual Interference on Performance Outcomes and Movement Patterns in Golf Skills. International journal of Sports Science \& Coaching. 2007;2(3):243-55.

34. Carter MJ, Ste-Marie DM. An interpolated activity during the knowledge-of-results delay interval eliminates the learning advantages of self-controlled feedback schedules. Psychological research. 2016.

35. Russell DM, Newell KM. How persistent and general is the contextual interference effect? Research quarterly for exercise and sport. 2007;78(4):318-27.

36.Zipp GP, Gentile AM. Practice Schedule and the Learning of Motor Skills in Children and Adults: Teaching Implications. Journal of College Teaching \& Learning. 2010;7(2):35-42.

37. Cross ES, Schmitt PJ, Grafton ST. Neural substrates of contextual interference during motor learning support a model of active preparation. Journal of cognitive neuroscience. 2007;19(11):1854-71.

38. Janelle CM, Barba DA, Frehlich SG, Tennant LK, Cauraugh JH. Maximizing performance feedback effectiveness through videotape replay and a self-controlled learning environment. Research quarterly for exercise and sport. 1997;68(4):269-79.

39. Carter MJ, Carlsen AN, Ste-Marie DM. Self-controlled feedback is effective if it is based on the learner's performance: a replication and extension of Chiviacowsky and Wulf (2005). Frontiers in Psychology. 2014;5:1325.

40. Chiviacowsky S, Wulf G, de Medeiros FL, Kaefer A, Wally R. Self-controlled feedback in 10-year-old children: higher feedback frequencies enhance learning. Research quarterly for exercise and sport. 2008;79(1):122-7. 